\title{
Treatment of bipolar depression with supraphysiologic doses of levothyroxine: a randomized, placebo-controlled study of comorbid anxiety symptoms
}

Maximilian Pilhatsch ${ }^{1 *}$, Thomas J Stamm², ${ }^{2,}$ Petra Stahl ${ }^{3}$, Ute Lewitzka ${ }^{1}$, Anne Berghöfer ${ }^{4}$, Cathrin Sauer ${ }^{1}$, Michael Gitlin ${ }^{5}$, Mark A. Frye ${ }^{6}$, Peter C. Whybrow ${ }^{5}$ and Michael Bauer ${ }^{1}$

\begin{abstract}
Background: Symptoms of anxiety co-occur in a variety of disorders including in depressive episodes of bipolar disorder and in patients with thyrotoxicosis. Treatment of refractory bipolar disorder with supraphysiologic doses of levothyroxine (L-T4) has been shown to improve the phenotypic expression of the disorder and is associated with an increase of circulating thyroid hormones. However, it might be associated with somatic and mental adverse effects. Here we report the investigation of the influence of treatment with supraphysiologic doses of L-T4 on symptoms of anxiety in patients with refractory bipolar depression.
\end{abstract}

Methods: Post-hoc analysis from a 6-week, multi-center, randomized, double-blind, placebo-controlled study of the effects of supraphysiologic L-T4 treatment on anxiety symptoms in bipolar depression. Anxiety symptoms were measured weekly with the Hamilton anxiety/somatization factor (HASF) score of the Hamilton Depression Rating Scale (HAMD) and the State- and Trait Anxiety Inventory (STAI).

Results: Treatment of both groups was associated with a significant reduction in anxiety symptoms $(p<0.001)$ with no statistical difference between groups ( $(\mathrm{TT}-4$ : from $5.9(\mathrm{SD}=2.0)$ at baseline to $3.7(\mathrm{SD}=2.4)$ at study end; placebo: from $6.1(S D=2.4)$ at baseline to $4.4(S D=2.8)$ at study end; $p=0.717)$. Severity of anxiety at baseline did not show a statistically significant correlation to the antidepressive effect of treatment with supraphysiologic doses of L-T4 $(p=0.811)$. Gender did not show an influence on the reduction of anxiety symptoms (females: from $5.6(S D=1.7)$ at baseline to $3.5(S D=2.4)$ at study end; males: from $6.1(S D=2.3)$ at baseline to $4.0(S D=2.4)$ at study end; $p=0.877)$.

Conclusions: This study failed to detect a difference in change of anxiety between bipolar depressed patients treated with supraphysiologic doses of L-T4 or placebo. Comorbid anxiety symptoms should not be considered a limitation for the administration of supraphysiologic doses of L-T4 refractory bipolar depressed patients.

Trial registration ClinicalTrials, ClinicalTrials.gov identifier: NCT01528839. Registered 2 June 2012-Retrospectively registered, https://clinicaltrials.gov/ct2/show/study/NCT01528839

\footnotetext{
*Correspondence: max.pilhatsch@uniklinikum-dresden.de

${ }^{1}$ Department of Psychiatry and Psychotherapy, Medical Faculty,

Universitätsklinikum Carl Gustav Carus, Technische Universität Dresden,

Fetscherstr. 74, 01307 Dresden, Germany

Full list of author information is available at the end of the article
} 


\section{Background}

Depression is the most difficult phase of bipolar disorder to treat (Bauer et al. 2018). Unfortunately, evidence from treatment trials in bipolar depression is sparse, and the role of antidepressants and their efficacy remains controversial (Gitlin 2018). Adjunctive treatment with thyroid hormones at supraphysiologic doses is one approach for treatment-refractory bipolar disorders for the acute depressive phase and maintenance treatment. While triiodothyronine (T3) demonstrated to be effective and safe in the treatment of affective disorders (Kelly and Lieberman 2009), the use of higher than normal, supplementary doses of levothyroxine (L-T4) has shown promise in several open-label studies, including for patients with rapid cycling (Bauer and Whybrow 1990), prophylaxisresistance (Baumgartner et al. 1994; Bauer et al. 2002b), and with acute refractory uni- or bipolar depression (Bauer et al. 1998, 2002a, 2005). These beneficial findings have been confirmed more recently in placebo-controlled studies (Stamm et al. 2014; Bauer et al. 2016; Walshaw et al. 2018): in the multicenter placebo-controlled study of Stamm et al. (2014), supraphysiologic doses of L-T4 were added to continuing treatment with mood stabilizer and/or antidepressant medication in 62 patients with bipolar depression. After 4 weeks but not at the study end at week 6 HAMD scores in the L-T4 group were significantly lower compared to the placebo group. However, the secondary analysis of women $(n=32)$ revealed a significant difference between groups in mean change in HAMD score in favor of the L-T4 treated group. Patients from a subsample $(\mathrm{N}=25)$ were enrolled to an imaging study assessing cerebral glucose metabolism with positron emission tomography (Bauer et al. 2016). Adjunctive treatment with supraphysiologic doses of L-T4 produced a significant decline in depression scores during the 6-week treatment, which was paralleled by restoration of metabolic activity in brain regions that are critically involved in the regulation of emotional processing and homeostasis. Recently, Walshaw et al. (2018) found that mood-stabilizing effects of adjunctive treatment with supraphysiologic doses of L-T4 were significantly higher compared to effects of T3 and placebo in 32 patients with treatment resistant rapid cycling.

Accordingly, treatment with supraphysiologic doses of L-T4 is recommended in several treatment guidelines for bipolar disorders (Grunze et al. 2010; Hirschfeld et al. 2010; Sachs et al. 2000; Yatham et al. 2018).

The hypothesis driving these studies was that increasing the availability of thyroid hormones to the brain changes the phenotypic expression of the disorder and is associated with improvement of mood that correlates with changes in brain metabolism in limbic areas (Bauer et al. 2005, 2016).
However, treatment with supraphysiological doses of thyroid hormones was erroneously classified as "thyrotoxic" and the patients treated as "hyperthyroid", which limited prescription by psychiatrists, as Kelly (2015) and Pilhatsch et al. (2018) pointed out. Meanwhile, a large number of studies indicate good tolerability showing lack of signs and symptoms of thyrotoxicity in patients with affective disorders who receiving supraphysiologic doses of thyroid hormones (Bauer et al.1998, 2005; Stamm et al. 2014; Bauer et al. 2016; Kelly 2015; Kelly et al. 2016). Monitoring of symptoms typically associated with thyrotoxicosis demonstrated no evidence for specific side effects: several prospective observational studies found no evidence for bone loss (Gyulai et al. 2001; Ricken et al. 2012), disturbed sleep (Kraemer et al. 2011) or increased risk for cardiovascular intolerance (Pilhatsch et al. 2018) during treatment with high dose L-T4. Moreover, studies of supraphysiologic doses of thyroid hormones used to prevent the reoccurrence of thyroid cancer did also not show a risk for bone loss or cardiovascular sequela (Kelly 2015; Kelly et al. 2016).

Epidemiological studies have revealed high rates of comorbidity between bipolar and anxiety disorders (Grande et al. 2016). Symptoms of anxiety are common during depressive episodes in bipolar disorder (Bauer et al. 2010; Grande et al. 2016). Likewise, episodic anxiety, frequently in association with subjective awareness of tachycardia or arrhythmia, is also a common symptom in thyrotoxic patients (Bauer et al. 2013). Indeed, some of these diffuse anxious and dysphoric feelings have been reported in normal subjects when given high doses of levothyroxine (L-T4) (Bauer et al. 2002a). Hence, it has been argued that treatment with supraphysiologic doses of L-T4 may lead-similar to thyrotoxicosis-to an increase of anxiety symptoms in bipolar depressed patients. To date, no study has focused on anxiety as a psychological side effect during treatment of L-T4.

We therefore identified a need to disentangle specific effects on depressive and comorbid anxiety symptoms during treatment of bipolar depression with supraphysiologic doses of L-T4. Moreover, we wanted to investigate if treatment with supraphysiologic doses of L-T4 is tolerated worse by patients with anxious depression. To achieve this goal, we assessed trait and state anxiety during the randomized, placebo controlled double-blind trial of adjunctive therapy with supraphysiologic L-T4 for bipolar depression (Stamm et al. 2014), mentioned above. Specifically, we investigated (i) whether supraphysiologic doses of L-T4 would lead to an increase of anxiety symptoms; (ii) whether severity of anxiety symptoms at baseline would moderate the antidepressive effect of treatment with supraphysiologic doses of L-T4; (iii) whether a gender difference can be identified regarding 
the effect of supraphysiologic doses of L-T4 and placebo on anxiety symptoms.

\section{Methods}

\section{Study design and sample}

This 6-week, multi-center, randomized, double-blind, placebo-controlled study was previously described in detail (Stamm et al. 2014). Participants were 18-65 years of age and diagnosed with bipolar disorder according to the Diagnostic and Statistical Manual of Mental Disorders, Fourth Edition (DSM-IV), currently in a depressive phase. They had failed to respond to at least 6 weeks of treatment with a mood stabilizer and/or antidepressant (see below) at standard doses according to the WFSBP guideline (Bauer et al. 2007). Serum levels of lithium were within therapeutic ranges $(0.5-0.8 \mathrm{mmol} / \mathrm{L})$ for at least 2 weeks before enrollment. Exclusion criteria were: (1) an axis I disorder other than bipolar disorder, (2) ultra-rapid cycling, defined as $\geq 12$ mood events in the previous year, (3) psychotic features, (4) a diagnosis of substance dependence or substance use (except for nicotine) over the previous year, (5) a clinically significant current medical illness, or (6) current or past thyroid disease or thyroid hormone treatment. Serum thyroid hormone [free thyroxine (fT4) and free triiodothyronine (fT3)] and thyroid-stimulating hormone (TSH) levels were required to fall within the normal reference range of the laboratory (fT4: 0.9-1.9 ng/dL; fT3: 2.6-5.1 ng/L; TSH 0.27-4.2 $\mathrm{mU} / \mathrm{L}$ ) in order to exclude subthreshold thyroid disease.

The study was carried out in accordance with the Declaration of Helsinki at six European centers. Ethical approval was obtained at each institution. All patients gave written informed consent. Please see Stamm et al. (2014) for a more comprehensive description of study design and procedures.

\section{Procedures and assessments}

Participants with a baseline score of at least 14 on the 17-item Hamilton Depression Rating Scale (HAMD, Hamilton 1960) and a Young Mania Rating Scale (Young et al. 1978) score below 13, entered the double-blind study phase. After randomization, they were given either a supraphysiologic dose of levothyroxine $(300 \mathrm{mcg} / \mathrm{d})$ or placebo as an add-on to their stable (at least 6-week) pre-treatment medication with a prophylactic and/or antidepressive medication. Affective symptoms were also assessed with the Montgomery-Asberg Depression Rating Scale (MADRS; Montgomery and Asberg 1979). Of 74 patients enrolled into the study, 62 were randomized to the study groups (L-T4 vs. placebo).

Anxiety symptoms were operationalized using the Hamilton anxiety/somatization factor (HASF) score of the HAMD scale and the State- and Trait-Anxiety
Inventory (STAI; Spielberger 1983). During the study, HAMD and STAI were assessed weekly from baseline until study end at week 6 .

\section{Statistical analyses}

Demographic and baseline clinical data were analyzed descriptively calculating means, standard deviations (SD), frequencies and contingency tables. Potential differences at baseline between the two treatment groups were examined using t-test and $\mathrm{Chi}^{2}$-test as appropriate. To assess the differences between groups concerning the outcomes we used t-tests, linear regression analysis as well as repeated-measures analysis-of-covariance (rmANCOVA) for analysis of the course of our outcomes over time. Outcome measures were HAMD, Hamilton anxiety/somatization factor (HASF) score, State-STAI and Trait-STAI. Independent variables were treatment (L-T4 vs. placebo), gender, and extent of comorbid anxiety symptoms at baseline (high vs. low). Age was included as covariate. Alpha error was adjusted to 5\%. Mauchly's Test of sphericity was used to test the assumption of sphericity. When sphericity was violated Huynh-Feldt corrections were performed. Missing follow-up data have been replaced by that subject's previously observed value, i.e. the last observation was carried forward. Statistics were done using SPSS-22 for Windows.

\section{Results}

\section{Demographical and clinical data}

Baseline comparisons of both demographic characteristics and clinical parameters such as severity of depressive symptoms (HAMD) and severity of anxiety symptoms (STAI state and trait) revealed no significant differences between treatment groups (Table 1). Age tended to be higher in controls $(\mathrm{p}=0.07)$ and was integrated as covariate in further analyses.

Comparisons of thyroid measurements showed that serum fT4 levels of female participants were significantly elevated at baseline compared to males $(0.9 \mathrm{ng} / \mathrm{dL}$ vs. $1.2 \mathrm{ng} / \mathrm{dL} ; \mathrm{p}=0.01$ ). As expected, treatment with L-T4 was associated with a significant increase of fT3 and fT4 and decrease of TSH compared to placebo at study end.

\section{Efficacy and tolerability outcomes}

The primary efficacy outcome of the main study has been previously reported (Stamm et al. 2014). In summary, mean change in HAMD score was larger in the L-T4 group compared to the placebo group during the 6-week study period. At week 4, but not at the endpoint of the study, depressive symptoms of patients treated with L-T4 significantly improved. In a post hoc analysis, a gender effect was found: female participants treated with L-T4 demonstrated a significant reduction of HAMD scores 
Table 1 Demographic and baseline clinical characteristics of participants with bipolar depression (intent-to-treat population)

\begin{tabular}{|c|c|c|c|}
\hline Item & Levothyroxine $(n=31)$ & Placebo $(n=31)$ & Difference \\
\hline Bipolar I/II, n & $15 / 16$ & $19 / 12$ & $C h i^{2}=1.042, d f=1, p=0.307$ \\
\hline Age, mean (SD), years & $41.8(12.8)$ & $48.0(14.2)$ & $T=1.8, d f=60, p=0.07$ \\
\hline Sex, male/female, n & $14 / 17$ & $16 / 15$ & $C h i^{2}=0.258, d f=1, p=0.611$ \\
\hline Total no. of mood episodes, mean (SD) & $8.0(7.8)$ & $9.5(9.2)$ & \\
\hline Duration of illness (mean), years & $12.3(9.7)$ & $18.1(15.1)$ & $T=1.694, d f=52, p=0.096$ \\
\hline Mean HASF score (SD); $n=62$ & $5.9(2.0)$ & $6.1(2.4)$ & $T=0.351, d f=60, p=0.727$ \\
\hline Mean State-STAI score (SD); $\mathrm{n}=61$ & $58.2(9.5)$ & $57.4(9.1)$ & $T=-0.347, d f=59, p=0.727$ \\
\hline Mean Trait-STAI score (SD); $\mathrm{n}=61$ & $58.4(11.4)$ & $57.9(9.9)$ & $T=0.189, d f=59, p=0.850$ \\
\hline HAMD baseline score, mean (SD) & $20.9(3.0)$ & $21.4(4.3)$ & $\mathrm{T}=0.549, \mathrm{df}=60, \mathrm{p}=0.585$ \\
\hline MADRS baseline score, mean (SD) & $28.8(5.6)$ & $30.3(6.0)$ & $T=1.031, d f=60, p=0.307$ \\
\hline
\end{tabular}

HASF Hamilton anxiety/somatization factor score, STAI State-Trait Anxiety Inventory, HAMD Hamilton Depression Rating Scale, MADRS Montgomery-Asberg Depression Rating Scale

compared to the placebo-treated group (Stamm et al. 2014).

With respect to tolerability no serious events occurred during the study. Regular assessments of ECG and body weight, measurements of blood pressure revealed normal results at baseline and indicated no significant changes during or after treatment. Monitoring of side effects typically associated with thyrotoxicosis with the "thyroid symptom list" (Bauer et al. 2002a) indicated no significant difference of such symptoms between groups at either the beginning or end of the study. However, three participants of the L-T4 group discontinued the study due to adverse events. In one of these cases mild thyrotoxicosis was diagnosed (Stamm et al. 2014).

\section{Anxiety measures}

We tested whether treatment with supraphysiologic doses of L-T4 leads to a change of comorbid anxiety symptoms compared to placebo. The mean HASF score at baseline was $5.9(\mathrm{SD}=2.0,95 \% \mathrm{CI}[5.1 ; 6.6])$ for the $\mathrm{L}-\mathrm{T} 4$ group and $6.1(\mathrm{SD}=2.4,95 \% \mathrm{CI}[5.2 ; 6.9])$ for the placebo group $(\mathrm{T}=0.351, \mathrm{df}=60, \mathrm{p}=0.727)$. As illustrated in Fig. 1a, treatment in this study was associated with a significant $(31.7 \%)$ decrease of anxiety symptoms (HASF score) from $6.0(\mathrm{SD}=2.2 ; 95 \% \mathrm{CI}[5.4 ; 6.5]$ at baseline to $4.1(\mathrm{SD}=2.6 ; 95 \% \mathrm{CI}[3.4 ; 4.7]$ after 6 weeks. $(\mathrm{T}=5.4$; $\mathrm{p}<0.001)$. In the LT-4 group, HASF score decreased by $37.3 \%$ (from baseline to $3.7(\mathrm{SD}=2.4 ; 95 \% \mathrm{CI}[2.9 ; 4.6])$ at week 6 . At study end there was no significant difference to the placebo group in which the HASF score decreased by $27.9 \%$ to $4.4(\mathrm{SD}=2.8,95 \% \mathrm{CI}[3.4 ; 5.4])$. The analyses with the STAI measures led to similar results (Fig. 1b, c).

Repeated measures ANCOVA revealed no significant main effect of treatment $(\mathrm{F}(1,59)=0.132, \mathrm{p}=0.717)$ or time $(\mathrm{F}(4.19,37.69)=0.501, \mathrm{p}=0.744$, Huynh-Feldt corrected). Age had no significant influence on the results. Interaction of time and treatment was also not significant $(\mathrm{F}(4.19,37.69)=0.826, \mathrm{p}=0.514$, HuynhFeldt corrected).

We further explored whether severity of anxiety symptoms at baseline would moderate the antidepressive effect of treatment with supraphysiologic doses of L-T4. Therefore, we examined the correlation between improvement of HAMD score from baseline to study end and the score for severity of anxiety at baseline, including HAMD baseline score as covariate. Linear regression analysis showed no statistical significance $(\mathrm{F}(2,28)=0.211, \mathrm{p}=0.811)$.

In the original publication, female patients benefitted more from treatment with supraphysiologic doses of L-T4 compared to male patients (Stamm et al. 2014). Therefore, we investigated whether a gender effect could be identified in the response of L-T4 treatment of anxiety symptoms. Seventeen female patients receiving L-T4 treatment had mean HASF scores of $5.6(\mathrm{SD}=1.7,95 \%$ CI $[4.8 ; 6.5]))$ at baseline indicating no significant difference to the 14 male participants $(6.1 ; \mathrm{SD}=2.3,95 \% \mathrm{CI}$ [4.8;7.5] $)(\mathrm{T}=0.688, \mathrm{df}=29, \mathrm{p}=0.497)$. At study end anxiety measures were reduced in both groups; the mean HASF score for female patients was $3.5(\mathrm{SD}=2.4,95 \%$ CI $[2.3 ; 4.8])$ and for male patients $4.0(\mathrm{SD}=2.4,95 \% \mathrm{CI}$ [2.6;5.4]). A repeated measures ANCOVA with gender as between subject factor revealed no main effect of time $(\mathrm{F}(4.5,17.3)=0.537, \mathrm{p}=0.730$, Huynh-Feldt corrected $)$ and no significant interaction of time and gender $(\mathrm{F}(4.5$, $17.3)=0.654, p=0.644$, Huynh-Feldt corrected). Gender as between-subject-factor had no significant influence on anxiety measures $(\mathrm{F}(1,28)=0.025, \mathrm{p}=0.877)$. These same analyses with State-STAI scores led to similar results (details not shown). 


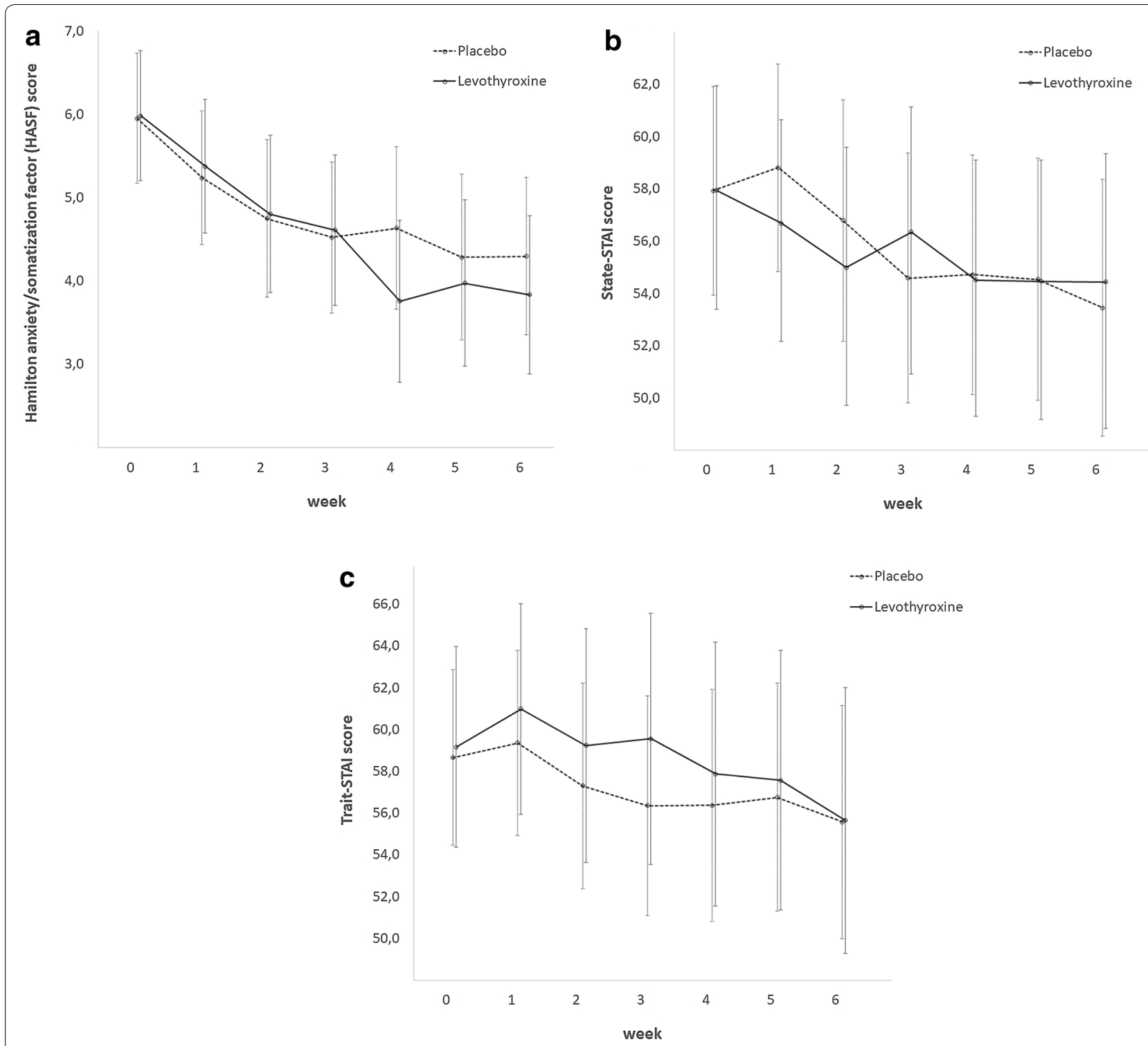

Fig. 1 Course of anxiety symptoms during 6-week treatment of bipolar depression in both treatment groups (L-T4 vs placebo). a Change of Hamilton anxiety/somatization factor (HASF) score. $\mathbf{b}$ Change of State-STAI score. $\mathbf{c}$ Change of Trait-STAI score

\section{Discussion}

In this 6-week randomized, double-blind, placebo-controlled trial adjunctive treatment with supraphysiologic doses of L-T4 in bipolar depression was associated with a significant reduction in anxiety symptoms. Compared to placebo, L-T4 treatment led to a numerical reduction of anxiety symptoms. However, this study failed to detect a statistic significant difference in change of anxiety between bipolar depressed patients treated with supraphysiologic doses of L-T4 or placebo.

The anxious-depressive subgroup of participants showed at least as good a response as the non-anxious-depressive patients. No gender differences were identified with regard to anxiety reduction during L-T4 augmentation.

Because treatment with supraphysiologic doses of L-T4 leads to an increase of thyroid hormone levels and thyrotoxicosis is associated with psychosis and anxiety, findings from this study are of clinical interest.

Results emphasize that the sequelae of people with thyrotoxicosis are different than those in patients with mood disorders resulting from treatment with supraphysiologic doses of T3 or L-T4 (Bauer et al. 2002a; Kelly 2015; Pilhatsch et al. 2018) suggesting a common entity between anxiety symptoms and depressive 
symptoms in bipolar disorders. Additionally, previous positron emission tomography (PET) studies have shown that anxiety disorders are associated with increased glucose metabolism in limbic brain structures such as hippocampus, parahippocampus and amygdala (Bisaga et al. 1998; Sakai et al. 2005). Another PET study indicated that comorbid anxiety symptoms have specific regional cerebral metabolic correlates in the same core limbic areas of patients with bipolar depression (Bauer et al. 2010). Given that these patterns of altered metabolism have specifically been restored by supraphysiologic treatment with L-T4 in a subsample of patients from this study (Bauer et al. 2016), our results underline that $\mathrm{L}-\mathrm{T} 4$ treatment is a valuable treatment option even in anxious depression from both a mechanistic and clinical perspective.

This study had several methodological limitations: it was a post hoc analysis; an observer-rated scale to measure anxiety specifically was not included (with the exemption of the HAMD scale containing anxiety items). Even if the sample appears relatively small, a post hoc power analysis on the repeated measures ANCOVA for the course of HASF score comparing LT-4 and placebo group shows a statistical power of $80 \%$.

In conclusion, based on data from this study supraphysiologic doses of L-T4 do not appear to increase anxiety symptoms, indicating that when treating patients with supraphysiologic doses of L-T4 there appears no reason to withhold it in patients with high severity of anxiety symptoms at baseline. Therefore, comorbid anxiety symptoms should advocate and not limit considering treatment with supraphysiologic doses of L-T4 as a valuable option for treatment refractory patients with bipolar depression.

\section{Acknowledgements}

The study was sponsored by the Stanley Medical Research Institute (Grant 02T-238 to M.B.). We acknowledge support by the Open Access Publication Funds of the SLUB/TU Dresden.

\section{Authors' contributions}

$M P, M B, T S, U L, A B$ and PW worked on the conception and design of the study. $M P, M B, P S, M G$ interpreted the results and drafted the manuscript. CS and PS performed the analyses. MP, UL, AB, MG and MF performed data collection, and interpretation of the findings. All authors read and approved the final manuscript.

\section{Funding}

This study was funded by the Stanley Medical Research Institute (Grant 02T238 to Dr Bauer).

\section{Availability of data and materials}

The data will not be shared or made publicly available. Informed consent for this was not sought from the study participants prior to the collection of data.

\section{Ethics approval and consent to participate}

All participating centers obtained approval from their Institutional Review Boards and all patients gave a written informed consent to participate in this study.

\section{Consent for publication}

Consent to publish has been obtained from the participants.

\section{Competing interests}

The authors declare that they have no competing interests.

\section{Author details}

${ }^{1}$ Department of Psychiatry and Psychotherapy, Medical Faculty, Universitätsklinikum Carl Gustav Carus, Technische Universität Dresden, Fetscherstr. 74, 01307 Dresden, Germany. ${ }^{2}$ Department of Psychiatry and Psychotherapy, Charité - Universitätsmedizin Berlin, Berlin, Germany. ${ }^{3}$ Psychiatrische Universitätsklinik der Charité, St. Hedwig-Krankenhaus, Berlin, Germany. ${ }^{4}$ Institute for Social Medicine, Epidemiology and Health Economics, Charité - Universitätsmedizin Berlin, Berlin, Germany. ${ }^{5}$ Department of Psychiatry and Biobehavioral Sciences, Semel Institute for Neuroscience and Human Behavior University of California Los Angeles (UCLA), Los Angeles, CA, USA. ${ }^{6}$ Department of Psychiatry \& Psychology, Mayo Clinic Depression Center, Mayo Clinic, Rochester, MN, USA. ${ }^{7}$ Department of Psychiatry, Psychotherapy and Psychosomatics, Brandenburg Medical School, Neuruppin, Germany.

Received: 10 May 2019 Accepted: 24 July 2019

Published online: 04 October 2019

\section{References}

Bauer MS, Whybrow PC. Rapid cycling bipolar affective disorder. II. Treatment of refractory rapid cycling with high-dose levothyroxine: a preliminary study. Arch Gen Psychiatry. 1990;47:435-40.

Bauer M, Hellweg R, Gräf KJ, Baumgartner A. Treatment of refractory depression with high-dose thyroxine. Neuropsychopharmacol. 1998;18:444-55.

Bauer M, Baur H, Berghöfer A, Ströhle A, Hellweg R, Müller-Oerlinghausen B, Baumgartner A. Effects of supraphysiological thyroxine administration in healthy controls and patients with depressive disorders. J Affect Disord. 2002a;68:285-94.

Bauer M, Berghöfer A, Bschor T, Baumgartner A, Kiesslinger U, Hellweg R, Adli M, Baethge C, Müller-Oerlinghausen B. Supraphysiological doses of L-thyroxine in the maintenance treatment of prophylaxis-resistant affective disorders. Neuropsychopharmacol. 2002b;27:620-8.

Bauer M, London ED, Rasgon N, Berman SM, Frye MA, Altshuler L, Mandelkern MA, Bramen J, Woods R, Mazziotta JC, Whybrow PC. Supraphysiological doses of levothyroxine alter regional cerebral metabolism and improve mood in women with bipolar depression. Mol Psychiatr. 2005;10:456-69.

Bauer M, Bschor T, Pfennig A, Whybrow PC, Angst J, Versiani M, et al. World Federation of Societies of Biological Psychiatry (WFSBP) guidelines for biological treatment of unipolar depressive disorders in primary care. World J Biol Psychiatry. 2007;8:67-104.

Bauer M, Berman SM, Schlagenhauf F, Voytek B, Rasgon N, Mandelkern MA Whybrow PC, London ED. Regional cerebral glucose metabolism and anxiety symptoms in bipolar depression: effects of levothyroxine. Psychiatry Res. 2010;181:71-6.

Bauer M, Samuels MH, Whybrow PC. Behavioral and psychiatric aspects of thyrotoxicosis. In: Braverman LE, Cooper DS, editors. Werner \& Ingbar's the thyroid. A fundamental and clinical text. 10th ed. Philadelphia: Wolters Kluwer Health, Lippincott Williams \& Wilkins; 2013. p. 475-80.

Bauer M, Berman S, Stamm T, Plotkin M, Adli M, Pilhatsch M, London ED, Hellemann GS, Whybrow PC, Schlagenhauf F. Levothyroxine effects on depressive symptoms and limbic glucose metabolism in bipolar disorder: a randomized, placebo-controlled positron emission tomography study. Mol Psychiatry. 2016;21:229-36.

Bauer M, Andreassen OA, Geddes JR, Vedel Kessing L, Lewitzka U, Schulze TG, Vieta E. Areas of uncertainties and unmet needs in bipolar disorders: clinical and research perspectives. Lancet Psychiatry. 2018;5(11):930-9.

Baumgartner A, Bauer M, Hellweg R. Treatment of intractable non-rapid cycling bipolar affective disorder with high-dose thyroxine: an open clinical trial. Neuropsychopharmacol. 1994;10:183-9.

Bisaga A, Katz JL, Antonini A, Wright CE, Margouleff C, Gorman JM, Eidelberg D. Cerebral glucose metabolism in women with panic disorder. Am J Psychiatry. 1998;155:1178-83.

Gitlin M. Antidepressants in bipolar depression: an enduring controversy. Int J Bipolar Disord. 2018;6(1):25. 
Grande I, Berk M, Birmaher B, Vieta E. Bipolar disorder. Lancet. 2016;387(10027):1561-72.

Grunze H, Vieta E, Goodwin GM, Bowden C, Licht RW, Moller HJ, Kasper S, Disorders, W.T.F.O.T.G.F.B. The World Federation of Societies of Biological Psychiatry (WFSBP) guidelines for the biological treatment of bipolar disorders: update 2010 on the treatment of acute bipolar depression. World J Biol Psychiatry. 2010;11:81-109.

Gyulai L, Bauer M, Garcia-Espana F, Hierholzer J, Baumgartner A, Berghöfer A, Whybrow PC. Bone mineral density in pre-and post-menopausal women with affective disorder treated with long-term L-thyroxine augmentation. J Affect Disord. 2001;66:185-91.

Hamilton M. A rating scale for depression. J Neurol Neurosurg Psychiatry. 1960;23:56-62.

Hirschfeld R, Bowden CL, Gitlin MJ, Keck PE, Suppes T, Thase ME, Wagner KD, Perlis RH. Practice guideline for the treatment of patients with bipolar disorder. 2nd ed. Philadelphia: American Psychiatric Association; 2010.

Kelly T. An examination of myth: a favorable cardiovascular risk-benefit analysis of high-dose thyroid for affective disorders. J Affect Disord. 2015;177:49-58

Kelly T, Lieberman DZ. The use of triiodothyronine as an augmentation agent in treatment-resistant bipolar II and bipolar disorder NOS. J Affect Disord. 2009;116:222-6.

Kelly T, Denmark L, Lieberman DZ. Elevated levels of circulating thyroid hormone do not cause the medical sequelae of hyperthyroidism. Prog Neuropsychopharmacol Biol Psychiatry. 2016;71:1-6.

Kraemer S, Danker-Hopfe H, Pilhatsch M, Bes F, Bauer M. Effects of supraphysiological doses of levothyroxine on sleep in healthy subjects: a prospective polysomnography study. J Thyroid Res. 2011;2011:420580.

Montgomery SA, Asberg M. A new depression scale designed to be sensitive to change. Br J Psychiatry. 1979;134(4):382-9.

Pilhatsch M, Berghöfer A, Mayer-Pelinski R, Berghöfer G, Ricken R, Möckel M, Kühnle Y, Sauer C, Whybrow PC, Bauer M. Long-term treatment with supraphysiologic doses of levothyroxine in treatment-refractory mood disorders - a prospective study of cardiovascular tolerability. J Affect Disord. 2018:238:213-7.

Ricken R, Bermpohl F, Schlattmann P, Bschor T, Adli M, Mönter N, Bauer M. Long-term treatment with supraphysiological doses of thyroid hormone in affective disorders — effects on bone mineral density. J Affect Disord. 2012;136:e89-94.

Sachs GS, Printz DJ, Kahn DA, Carpenter D, Docherty JP. The Expert Consensus Guideline Series: Medication Treatment of Bipolar Disorder 2000. Postgrad Med. 2000; Spec No:1-104.

Sakai Y, Kumano H, Nishikawa M, Sakano Y, Kaiya H, Imabayashi E, Ohnishi T, Matsuda H, Yasuda A, Sato A, Diksic M, Kuboki T. Cerebral glucose metabolism associated with a fear network in panic disorder. NeuroReport. 2005;16:927-31.

Spielberger C. Manual for the State Trait Anxiety Inventory. Santa Clara: PaloAlto; 1983.

Stamm TJ, Lewitzka U, Sauer C, Pilhatsch M, Smolka MN, Koeberle U, Adli M, Ricken R, Scherk H, Frye MA, Juckel G, Assion HJ, Gitlin M, Whybrow PC, Bauer M. Supraphysiologic doses of levothyroxine as adjunctive therapy in bipolar depression: a randomized, double-blind, placebo-controlled study. J Clin Psychiatry. 2014;75:162-8.

Walshaw PD, Gyulai L, Bauer M, Bauer MS, Calimlim B, Sugar CA, Whybrow PC. Adjunctive thyroid hormone treatment in rapid cycling bipolar disorder: a double-blind placebo-controlled trial of levothyroxine (L-T4) and triiodothyronine (T3). Bipolar Disord. 2018;20(7):594-603.

Yatham LN, Kennedy SH, Parikh SV, Schaffer A, Bond DJ, Frey BN, Sharma V, Goldstein BI, Rej S, Beaulieu S, Alda M, MacQueen G, Milev RV, Ravindran A, O'Donovan C, McIntosh D, Lam RW, Vazquez G, Kapczinski F, McIntyre RS, Kozicky J, Kanba S, Lafer B, Suppes T, Calabrese JR, Vieta E, Malhi G, Post RM, Berk M. Canadian Network for Mood and Anxiety Treatments (CANMAT) and International Society for Bipolar Disorders (ISBD) 2018 guidelines for the management of patients with bipolar disorder. Bipolar Disord. 2018;20:97-170.

Young RC, Biggs JT, Ziegler VE, Meyer DA. A rating scale for mania: reliability, validity and sensitivity. Br J Psychiatry. 1978;133:429-35.

\section{Publisher's Note}

Springer Nature remains neutral with regard to jurisdictional claims in published maps and institutional affiliations.

\section{Submit your manuscript to a SpringerOpen ${ }^{\circ}$ journal and benefit from:}

- Convenient online submission

- Rigorous peer review

- Open access: articles freely available online

- High visibility within the field

- Retaining the copyright to your article

Submit your next manuscript at $\boldsymbol{\nabla}$ springeropen.com 